\title{
Politics of Patents
}

\section{Researching, Making and Wearing Alternative Histories of Clothing Inventions}

Kat Jungnickel

On 6 December 1895, Alice Louisa Bygrave registered an English patent for "Improvements in Ladies' Cycling Skirts". The patent tells us she was a dressmaker living in South London, at No. 13 Canterbury Road, Brixton. She explains the objective of the invention is "to provide a skirt proper to wear when either on or off the machine". While the design makes use of "an ordinary skirt" and "ordinary knickerbockers", the novelty lies in the infrastructure of the garments. Inside the seams, waistband and hems is a unique pulley system (see Figure 1). Through a careful combination of "cords", "suitable guides" and "weights", Bygrave's innovation operates by "raising the skirt before and behind to a sufficient height".

Figure 1: Alice Bygrave's 1895 patent for “Improvements in Ladies' Cycling Skirts” (image used with permission of the European Patent Office [www.epo.org]).

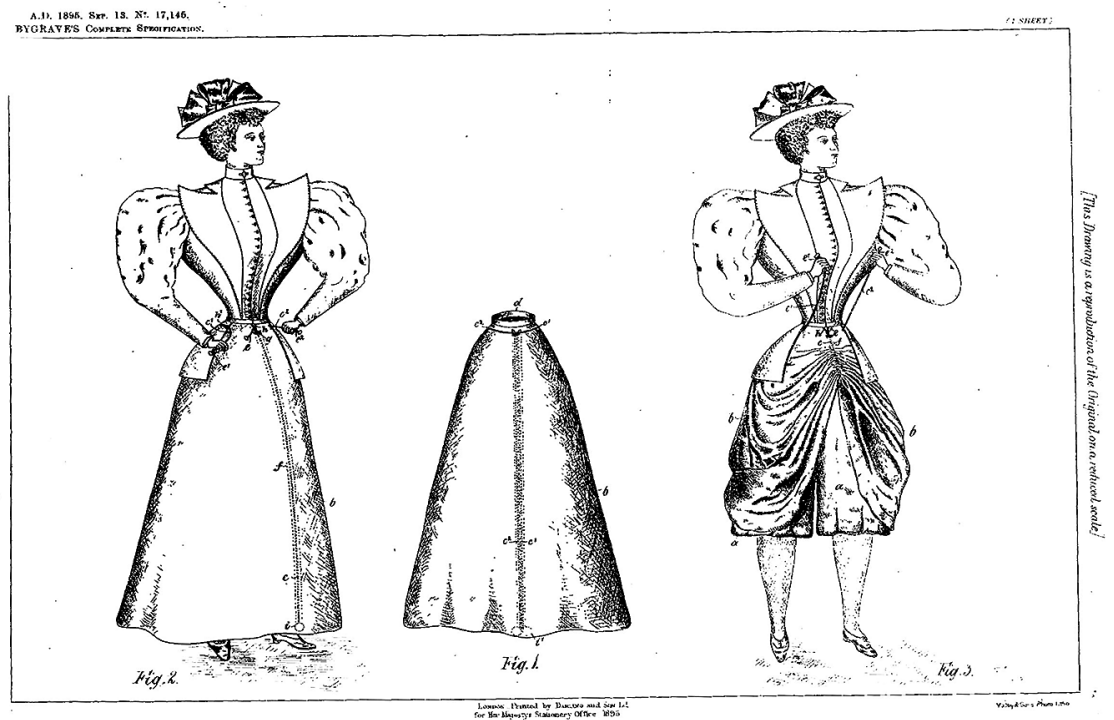

Bygrave's convertible cycling skirt is remarkable. Her carefully concealed design gathers the material at the front and rear of the skirt, up and out of the way of moving machinery - bicycle pedals, chain and wheels - to enable the wearer to 
secretly switch from walking skirt to safe and comfortable cycling costume when needed. Sewn into the seams of the skirt, this convertible system also deliberately hides her cycling intentions from parts of society who might otherwise hurl abuse or stones at "the hapless women who dared to reveal the secret that she had two legs” (Marshall 1899: 40). Bygrave's invention, and others like it, carved out means and space for women to not only imagine, but also furnish themselves as independent, mobile citizens in new social, political and economic worlds.

This is one of the many fascinating invention stories from research conducted in the European Patent Office digital archives. I have been exploring what historic clothing inventions and inventors can tell us about the changing nature of citizenship over time in two projects: "Bikes and Bloomers: Victorian Inventors and Their Extraordinary Cyclewear" and "Politics of Patents (POP): Re-imagining Citizenship via Clothing Inventions 1820-2020”. This research takes a gender/ queer, decolonial and science and technology studies (STS)/feminist technoscience approach to the study of clothing inventions, embodied knowledges and the history of clothing as wearable technology.

My research team and I approach the patent archive to ask: How are citizens made and re-imagined through clothing inventions? From an STS lens, this means viewing clothing as a sociopolitical device that enables, constrains and organises bodies in different ways. We explore clothing inventions as "acts of citizenship" (Isin/Neilson 2008), which opens up possibilities of studying how people socially, spatially, materially, performatively and economically "do" and "make" citizenship in terms of claiming space, interrupting order or otherwise engaging in or attempting to shape social and political worlds on a more daily basis. In the POPLab, we are investigating how inventors create new forms of clothing that resist, subvert or disrupt social and political norms and beliefs and in the process bring new expressions of citizenship into being.

Historic patents provide a valuable record and rich source of alternative sociotechnical data. Patents are problem-making and problem-solving devices. Inventors explain what concerned them and how to fix it, materially and technically. In the process, they reveal glimpses into the sociocultural context of the time, the politics of clothing, historic maker communities and feminist cultures of invention.

Patents get us closer to the experience of a range of inventors far beyond that of popular discourse. We learn of smaller and lesser-known inventions and get beyond the usual focus on "heroes, big men, important organisations, or major projects" (Law 1990: 12) and take into account more diverse and often radical contributions of women and other marginalised people more commonly silenced or systematically erased from the histories of technology. Zorina Khan writes: "Patent records present a valuable perspective on female inventive activity and market participation in an era when marriage meant the virtual 'invisibility' of married women in terms of objective data" (2000: 163).

Patent archives are critically important sites of data because they provide evidence of women, and other marginalised groups, actively driving change. They 


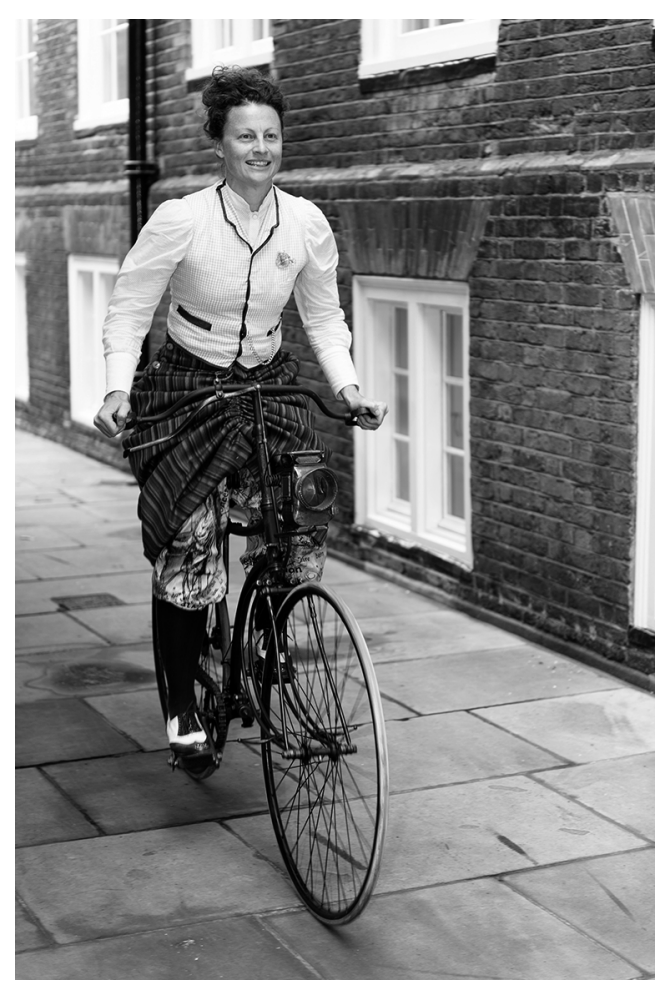

Figure 2: Reconstruction of Alice Louisa Bygrave's 1895 patent for "Improvements in Ladies' Cycling Skirts" (image used with permission of Charlotte Barnes www.charlottebarnes.co.uk).

were not passively waiting for the situation to be resolved. They were not simply buffeted by social waves of change. Rather, many were actively attempting to drive it. By making and declaring their designs in public, they became important actors in socio-technical change - legitimising women's cycle wear as valid inventions, their bodies as rightful actors in mobile public space and claiming a place for women in politics and business.

Like many inventions, Bygrave's convertible skirt is fascinating on paper. It is even more remarkable when transformed (back) into wearable technology (see Figure 2). An integral part of our methodological approach involves analysing patents via text and image and also materially and physically. We follow inventors' instructions step-by-step and stitch-by-stitch to reconstruct their inventions. In previous work, I have argued that this approach enables us to interrogate clothing patents as three-dimensional dynamic knowledge objects. In the process, we participate in choreographies of ideas, materials and practice; party to the productive mess, mistakes and mishaps of making. There is an intimacy in sewing and wearing the clothes of others, especially when the owners of these garments lived over a century ago. Distance in time and space is diminished as we get up close and into the research in new ways (Jungnickel 2018, 2020). This is especially important with dynamic wearable technologies like these, designed to switch from one modality to another and to be worn on lively moving bodies. 
There are many striking features of Bygrave's convertible skirt, not least the fact that its inventiveness was hidden in plain sight. Her design deliberately concealed the wearer's cycling intentions, enabling her to cycle safely when desired, while also offering some protection from the daily threat of harassment. These were very real experiences for early Victorian women cyclists. Convertible cycle wear inventors reveal to us not only how individuals managed to continue to participate in a much-loved activity, but also how collectively their radically dressed bodies played a small but nevertheless critical role in intervening in social norms and legal systems around mobility and public space, helping to legitimise new mobile forms of gendered citizenship in public place.

\section{Acknowledgements}

Politics of Patents (POP) is a 5-year research project (2019-2024) funded by a European Research Council consolidator grant \#819458 (www.politicsofpatents.org).

The article also references images and research undertaken in Bikes and Bloomers, part of the Transmissions and Entanglements: Making, Curating and Representing Knowledge project supported by an Economic and Social Research Council Knowledge Exchange grant and Intel Corporation (ES/Koo8०48/1) (www. bikesandbloomers.com).

\section{References}

Isin, E./Neilson, G. (eds.) (2008): Acts of Citizenship. London \& New York: Zed Books.

Jungnickel, K. (2018): Bikes and Bloomers: Victorian Women Inventors and Their Extraordinary Cycle Wear. London: Goldsmiths Press.

Jungnickel, K. (ed.) (2020): “Making and Wearing.” In: Transmissions: Critical Tactics for Making and Communicating Research. Cambridge, MA: MIT Press, pp. 66-88.

Khan, B.Z. (2000): “'Not for Ornament': Patenting Activity by Nineteenth-Century Women Inventors.” Journal of Interdisciplinary History 31(2), pp. 159-195.

Law, J. (1990): "Introduction: Monsters, Machines and Sociotechnical Relations." The Sociological Review 38(S1), pp. 1-23.

Marshall, I. (1899): “Correspondence.” The Rational Dress Gazette: Organ of the Rational Dress League, No. 10. Accessed at the University of Hull, UK. 